\title{
Dual Antiplatelet Therapy (DAPT) in Coronary Artery Disease: A Daunting Dilemma
}

\author{
Suryateja Chaturvedula ${ }^{1}$ M.D, Daniel J. Diver ${ }^{1,2}$, M.D., Aseem Vashist ${ }^{1,2,3}$, M.D.
}

University of Connecticut School of Medicine, Farmington $\mathrm{CT}^{1}$; Hoffman Heart and Vascular Institute at St

Francis Hospital and Medical Center, Hartford, $\mathrm{CT}^{2}$; VACT Healthcare System, West Haven, $\mathrm{CT}^{3}$.

\section{Address for Correspondence:}

\author{
Aseem Vashist, M.D. \\ Associate Professor of Medicine, \\ University of Connecticut School of Medicine, \\ Hoffman Heart and Vascular Institute, \\ Hartford, CT \\ E-mail: vashist@uchc.edu
}

\begin{abstract}
Percutaneous coronary intervention $(\mathrm{PCl})$ with stenting for the treatment of acute coronary syndrome(ACS) is the contemporary standard of care. Such treatment is followed by Dual anti-platelet therapy(DAPT) comprising of aspirin and a P2Y12 inhibitor. The efficacy of this therapy has been well established but the optimal duration of DAPT remains elusive, and has thus far attracted a prodigious deal of scientific attention. Decision regarding DAPT duration can be challenging clinically in the modern era with the evolution of newer stents, more potent antiplatelet agents and novel anticoagulant drugs in addition to an older patient population with multiple comorbidities. Major societal guidelines have emphasized comprehensive assessment of ischemic and bleeding risk, in turn recommending individualization of DAPT duration, thus encouraging "shared decision making". The following review is aimed at critically evaluating the available evidence to help make these crucial clinical decisions regarding duration of DAPT and triple therapy.
\end{abstract}

Keywords: dual anti platelet therapy; acute coronary syndrome; stents 


\section{Introduction:}

Coronary revascularization with percutaneous intervention $(\mathrm{PCl})$ is currently the standard of care in the treatment of patients with acute coronary syndrome(ACS), and has become one of the most frequently performed therapeutic procedures in Medicine ${ }^{1}$. Dual anti-platelet therapy (DAPT) comprising of aspirin and a $\mathrm{P} 2 \mathrm{Y} 12$ inhibitor is one of the most commonly prescribed therapies in cardiovascular medicine. The optimal duration of DAPT after stent implantation has been a matter of intense debate and has attracted a great deal of scientific attention. As we celebrate the various advances in the techniques and technology of transcatheter therapeutics in this $40^{\text {th }}$ year of Interventional Cardiology, optimal duration of DAPT continue to be elusive. Most recent transatlantic guidelines have called for the comprehensive assessment of ischemic and bleeding risks thus emphasizing individualization of $\mathrm{DAPT}^{2,3}$. The following review is aimed at critically evaluating the available evidence to help make crucial clinical decisions regarding duration of DAPT and triple therapy.

\section{Evolution of $\mathrm{PCl}$ :}

Careful examination of the history of PCI provides important insights into the evolution of DAPT ${ }^{4}$. Initial enthusiasm of angioplasty as an alternative to bypass surgery was stifled by the limitations owing to abrupt vessel closure due to recoil, dissections and restenosis thus leading to development of stents to offer luminal integrity without compromising safety ${ }^{5,6}$. Bare metal stents(BMS) were affected by restenosis with a need for repeat revascularization in up to one third of the patients ${ }^{7}$ besides lethal early stent thrombosis (EST; $<30$ days) ${ }^{8}$. Drug eluting stents (DES) were designed and in their first iteration, they were clearly superior to $\mathrm{BMS}$ in reducing restenosis and rates of repeat revascularizations ${ }^{9-12}$. There was a trend for increased late stent thrombosis(LST) (>30days <1 year) and very late stent thrombosis (VLST) (>1 year), and this was felt to be multifactorial. Subsequently, second generation DES endowed with better biocompatibility, thinner platforms ensured improved vessel healing ${ }^{13}$. In a meta-analysis of four randomized controlled trials(RCTs) comparing Everolimus eluting stents (second generation DES) vs Paclitaxel eluting stents (first generation DES) a significant reduction in stent thrombosis (ST) was noted $(0.7 \% \text { vs } 2.3 \% \text {; odds ratio[OR]: } 0.32 ; p<0.00001)^{14}$. To modify the interference with vasoregulation, chronic inflammation, neoatherosclerosis and device fractures, in turn leading to target-lesion failure(TLF) ${ }^{15,16}$ bioresorbable vascular scaffolds(BVS) ${ }^{16-18}$ were introduced. However, there is a lot to desire with the current BVS technology and the most recent recommendations dissuade providers from their preferential use over DES ${ }^{19}$. Till good quality evidence emerges from RCTs against contemporary DES powered for a surrogate end point of clinical efficacy, BVS is unlikely to be in routine use.

\section{Rationale for DAPT:}

The idea of antiplatelet therapy in reducing thrombosis following $\mathrm{PCl}$ was kindled three decades ago ${ }^{20}$. However, use of DAPT became a standard in BMS era to reduce the rates of EST ${ }^{21}$. DAPT was reserved for 3-6 months for the use of first generation DES to prevent ST and to ensure endothelialization. The DAPT duration in the earlier times of first generation DES era was up to 6 months ${ }^{9,10,22}$. With the realization of higher thrombotic milieu in the first year with these stents, especially with interruption of DAPT ${ }^{23,24}$, consensus based guidelines recommended prolonging DAPT to up to 12 months ${ }^{25}$. This philosophy was reinforced by the observed benefits of such therapy in prevention of atherothrombosis of non-stented segments in coronary vasculature ${ }^{26}$. With the ubiquitous use of second generation DES with lower rates of ST, and evidence from multiple RCTs supporting shorter DAPT, guidelines proposed optimal DAPT duration of 6-12 months ${ }^{2,27}$. Subsequently, two large RCTs demonstrated benefit in stent related and unrelated ischemic events at the cost of increased bleeding ${ }^{28,29}$ thus re-igniting the short vs long debate. The most recent iteration of guidelines takes cognizance of all these data and call for shared decision making and individualizing DAPT ${ }^{30}$. 


\section{Antiplatelet agents and their landmark trials:}

Table1 enumerates the cardinal pharmacological properties of various $\mathrm{P} 2 \mathrm{Y} 12$ inhibitors which are an essential component of DAPT. Importantly, the differences in the recommended periods of discontinuation of these agents in the lead up to non-emergent surgery should be noted.

\section{TABLE 1 Comparative properties involving oral P2Y12 inhibitors}

$\mathrm{N} / \mathrm{A}=$ not applicable

- In patients with ACS previously exposed to clopidogrel, switching to ticagrelor is recommended early after hospital admission at a loading dose of $180 \mathrm{mg}$ irrespective of timing and loading dose of clopidogrel, unless contraindicated $(\mathrm{IB})^{30}$

- $\quad$ All other switching between P2Y12 inhibitors may be considered in cases of side effects/intolerance(IIb-C $)^{30}$

While an exhaustive review of all the trials is beyond the scope of this review, Table 2 summarizes the evidence leading to the inception of various antiplatelet agents in the treatment of coronary artery disease (CAD). The outcome measures observed in these trials highlight the ability of these drugs in improving cardiovascular outcomes, albeit at the cost of increasing bleeding.

\section{TABLE 2 Landmark trials of antiplatelet agents}

MACCE = major adverse cardiovascular and cerebrovascular events; $\mathbf{I C H}=$ Intracranial hemorrhage

ISIS-2 $^{31}=$ Second International Study of Infarct Survival Collaborative Group; CAPRIE ${ }^{32}=$ A randomized, blinded, trial of Clopidogrel versus Aspirin in Patients at Risk of Ischemic Events; CURE ${ }^{33}=$ Clopidogrel in Unstable angina to prevent Recurrent Events; PCICURE $^{34}=$ Effects of pretreatment with clopidogrel and aspirin followed by long-term therapy in patients undergoing percutaneous coronary intervention; $\mathbf{C R E D O}^{35}=$ The Clopidogrel for the Reduction of Events During Observation; TRITON-TIMI $\mathbf{3 8}^{36}=$ TRial to assess Improvement in Therapeutic Outcomes by optimizing platelet Inhibition with Prasugrel-Thrombolysis in Myocardial Infarction, PLATO $^{37}=$ Platelet inhibition And patient Outcomes; TRILOGY ACS ${ }^{38}=$ The TaRgeted platelet Inhibition to cLarify the Optimal strateGy to medicallY manage Acute Coronary Syndromes; CHAMPION PHOENIX ${ }^{39}=$ Effect of platelet inhibition with cangrelor during $\mathrm{PCl}$ on ischemic events; TRACER $^{40}=$ Thrombin-Receptor Antagonist for Clinical Event Reduction.

The benefits of DAPT in a RCT setting were first seen in the CURE ${ }^{33}$ trial in which combination of aspirin and clopidogrel in comparison with aspirin monotherapy was assessed in patients undergoing $\mathrm{PCl}$ in myocardial infarction(MI) without ST elevation. 12,562 patients were enrolled and at mean follow up of 9 months, DAPT was associated with a significant reduction in the composite primary end point of cardiovascular mortality (CVM), non-fatal MI, or stroke [9.3\% vs. 11.4\%, Relative risk(RR):0.80; $p<0.001 ;$ Number needed to treat $(\mathrm{NNT})=48]$. However, this came at a cost of increased rate of major bleeding [3.7\% vs. 2.7\%, RR: 1.38; $\mathrm{p}=0.001]$. This trial was instrumental in establishing the 12 months of DAPT as standard of care in the treatment of ACS patients.

In TRITON-TIMI $\mathbf{3 8}^{36}$ trial, 13,608 patients with ACS awaiting PCI were randomized to either prasugrel or clopidogrel in addition to the usual care. At mean follow-up of 14.5 months, composite primary end point of CVM, non-fatal MI, or stroke was significantly lower in the prasugrel group [9.9\% vs. $12.1 \%$ (HR:0.81; $p<0.001$; NNT=46]. These benefits came at the cost of increased risk of bleeding. Major bleeding was higher with the use of prasugrel group vs. clopidogrel $(2.4 \%$ vs. $1.8 \%$, HR $1.32 ; p=0.03)$. Also, greater in the prasugrel group 
was the rate of life-threatening bleeding ( $1.4 \%$ vs. $0.9 \% ; p=0.01)$, including nonfatal bleeding ( $1.1 \%$ vs. $0.9 \%$; HR 1.25; $p=0.23)$ and fatal bleeding $(0.4 \%$ vs. $0.1 \% ; P=0.002)$. There was no significant difference in either CVM or all-cause mortality (ACM). Interestingly, the benefits appeared within days from randomization and persisted beyond the first year. In the sub-group analysis of patients with ST elevation MI(STEMI), there was an even greater benefit in the primary outcome (6.5\% vs. 9.5\%; HR: 0.68; $p=0.0017)$ without the incremental bleeding risk ${ }^{41}$. In the subsequent TRILOGY ACS trial ${ }^{38}$, there was no significant risk reduction of primary endpoint with the use of prasugrel in patients with unstable angina(UA) and non-ST elevation myocardial infarction(NSTEMI) treated without revascularization.

In one of the largest RCTs', Ticagrelor was compared to clopidogrel in the PLATO trial ${ }^{37}$. PLATO randomized 18,624 patients with ACS (37.5\% presenting with STEMI) were randomized to ticagrelor or clopidogrel in addition to standard care. At 12 months, ticagrelor group had lower composite primary outcome of CVM, MI, or stroke $(9.8 \%$ vs. $11.7 \%$; HR: $0.77-0.92 ; p<0.001)$ and there was insignificant increase in major bleeding (11.6\% vs. $11.2 \%$; HR: $1.04 ; p=0.43)$. A reduction in vascular mortality ( $4 \%$ vs. $5.1 \% ; H R: 0.79 ; p<0.001)$ and ACM (4.5\% vs. 5.9\%; HR: 0.78; $p<0.001)$ were also noted. However, the reduction in stroke was statistically not significant (1.5\% vs. $1.3 \%$; HR: $1.17 ; p=0.22)$. Ticagrelor is the only antiplatelet agent shown to decrease the ACM compared to clopidogrel though given the hierarchical statistical design of this study, the significance of this finding is attenuated.

The real life experience of ticagrelor was evaluated in SWEDEHEART registry ${ }^{42}$. This non-randomized prospective cohort study of 45,073 ACS patients in Sweden demonstrated amplified benefits of ticagrelor in comparison with clopidogrel. The composite primary outcome of ACM, re-admission with myocardial infarction (MI) or stroke was lower with ticagrelor group [11.7 vs. 22.3\%, adjusted HR 0.85]. In a subset of patients undergoing $\mathrm{PCl}$ on ticagrelor, the $\mathrm{PCl}$-related in-hospital bleeding was higher [3.7 vs. 2.7\%, adjusted OR: 1.57 (1.30-1.90)]. This registry data certainly corroborates the evidence from PLATO trial but some major differences are noteworthy, as evidenced by the mean age of patients in the present study being 8 years higher (70 vs. 62 years) and a higher proportion of patients with history of stroke $(10.8 \%$ vs. $3.9 \%)$ and heart failure $(10.3 \%$ vs. $5.6 \%)$.

\section{Duration of DAPT:}

Traditionally 12 months of DAPT duration has been considered as the standard with 3months and 6 months of DAPT representing short DAPT (S-DAPT) and $>12$ months representing longer DAPT (L-DAPT) durations. The conception of S-DAPT was to reduce bleeding without compromising the safety and efficacy of PCl, while LDAPT was tested with a hope to improve stent related and stent unrelated ischemic (Atherothormbotic) events. With the development of better stent platforms amounting to reduction in rates of ST and restenosis paralleled by development of potent antiplatelet agents, the "optimal" duration of DAPT has been extensively evaluated but still remains elusive. This constant dualistic debate of "short" vs "long" has certainly lead to significant uncertainty and confusion among the treating providers. Some observers have recommended an end to such a dogmatic approach laced with academic debates, and emphasized shared decision making and individualization of therapy ${ }^{43}$.

\subsection{EVIDENCE ON DAPT DURATION}

Till date there have been several RCTs' and several meta-analyses of these trials to evaluate for optimal duration of DAPT. At the outset, it is crucial to note the several limitations to these trials including but not limited to flaws in design ultimately leading to lack of power in detecting difference in hard endpoints, varying patient and lesion complexity, diverse clinical settings, low event rates, different times of randomization, slow 
enrollment, dissimilar endpoints, differential use of stents (BMS; first vs second generation DES) thus making comparable interpretation difficult and yielding inconsistent results ${ }^{44}$.

\section{2a RCT with S-DAPT}

There have been 12 RCTs' using S-DAPT to determine its relative efficacy in preventing major adverse cardiovascular and cerebrovascular events (MACCE) including ST, and to determine the relative safety of such DAPT duration for major bleeding in comparison with standard or L-DAPT. Unfortunately, none of these trials were independently powered to evaluate the rates of safety end point of ST which is infrequent.

\section{2b S-DAPT vs. standard DAPT duration}

The hypothesis of non-inferiority of S-DAPT to standard care was tested in 9 RCTs. These are comparatively summarized in Table 3.

TABLE 3 Comparative features of randomized controlled trials(RCTs) for short DAPT(S-DAPT) Vs. standard DAPT

ACS= acute coronary syndrome; $\mathbf{D M}=$ diabetes mellitus; $\mathbf{1 G}=$ first generation; $\mathbf{2 G}=$ second generation; $\mathbf{M I}=$ myocardial infarction; $\mathbf{T V R}=$ target vessel revascularization; $\mathbf{p}_{\mathrm{ni}}=\mathrm{p}$ value for non-inferiority; $\mathbf{S T}=$ stent thrombosis; $\mathbf{T I M I}=$ thrombolysis in $\mathrm{myocardial}$ infarction; $\mathbf{B A R C}=$ bleeding academic research consortium; $\mathbf{T L R}=$ target lesion revascularization

EXCELLENT ${ }^{45}=$ Six-month versus 12 -month dual antiplatelet therapy after implantation of drug-eluting stents: the Efficacy of Xience/Promus versus Cypher to REduce Late Loss After Stenting randomized, multicenter study; RESET ${ }^{46}=$ REal Safety and $^{2}$ Efficacy of 3-month dual antiplatelet Therapy following Endeavor zotarolimus-eluting stent implantation; OPTIMIZE ${ }^{47}=$ Three vS twelve months of dual antiplatelet therapy after zotarolimus-eluting stents; SECURITY ${ }^{48}=$ Second-generation drug-eluting stent implantation followed by 6- versus 12 -month dual antiplatelet therapy; ISAR-SAFE ${ }^{49}=$ Intracoronary Stenting and Antithrombotic Regimen: Safety and Efficacy of 6 months' DAPT after DES; I LOVE IT ${ }^{50}=$ a randomized, double-blind, placebo-controlled trial of 6 vs. 12 months of clopidogrel therapy after drug-eluting stenting; IVUS XPL ${ }^{51}=6$-Month Versus 12-Month Dual-Antiplatelet Therapy Following Long Everolimus-Eluting Stent Implantation; DAPT-STEMI ${ }^{52}=$ A prospective, randomized, open-label trial of 6month versus 12-month dual antiplatelet therapy after drug-eluting stent implantation in ST-elevation myocardial infarction; REDUCE $^{53}=$ Randomized evaluation of short-term dual antiplatelet therapy in patients with acute coronary syndrome treated with the COMBO dual therapy stent

It is imperative to note that, patients were randomized to DAPT duration at the time of stent implantation in all trials except in ISAR-SAFE ${ }^{49}$. There is significant heterogeneity among these trials with regards to the enrollment of patients with ACS, diabetes, and the type of stent used.

The ISAR-SAFE ${ }^{49}$ trial was actually designed to enroll 6000 patients with a non-inferiority hypothesis. However, it was prematurely terminated after enrolling 4000 patients' due to slow enrollment but still achieved noninferiority. These patients who had undergone PCI with DES were randomized at 6 months to interrupt or continue 12 months of DAPT. There was a fair representation of patients with ACS (40\%), with $10 \%$ suffering from STEMI and $30 \%$ with multivessel CAD. Second generation DES were predominantly used (89\%) and mostly used in treatment of single lesion (63\%). Primary composite outcome of death, MI, ST, stroke, or thrombolysis in myocardial infarction (TIMI) major bleeding occurred in 1.5\% of patients on S-DAPT and $1.6 \%$ with standard DAPT ( $p_{\mathrm{ni}}<0.001$ with predefined noninferiority margin of $2 \%$ ). Both groups had similar rates of TIMI major bleeding.

Two trials with a novel approach were presented at Transcatheter Cardiovascular Therapeutics meeting (TCT 2017), Denver, CO, November 1, 2017. In the DAPT-STEMI trial, patients with STEMI and undergoing primary 
$\mathrm{PCl}$ with a second-generation DES (zotarolimus-eluting stent [ZES]) were randomized in a 1:1 fashion to receive either 6 months $(n=433)$ or 12 months of DAPT $(n=437)$ to assess the safety and efficacy of such DAPT durations. Importantly, patients without any events in the first 6 months [MI, ST, target vessel failure (TVF) or target lesion failure (TLF), or stroke/bleeding requiring DAPT discontinuation] were included in the analysis. Patients requiring left main coronary artery (LMCA) intervention were excluded. All three contemporary P2Y12 agents were used (clopidogrel: 42\%, prasugrel: 30\%, ticagrelor: 29\%).The primary outcome, ACM, MI, revascularization, stroke, and TIMI major bleeding at 18 months was lower in 6-month vs. 12-month DAPT $\left(4.8 \%\right.$ vs. $\left.6.6 \%, \mathrm{p}_{\mathrm{ni}}=0.004\right)$. Although, this 2 -year outcome data establishes non-inferiority of S-DAPT in STEMI patients, this trial was not powered to evaluate for individual safety end points. Long-term data would be crucial before such short DAPT duration is adapted into clinical practice in the treatment of ACS.

In the REDUCE trial, 3-Month vs. 12-Month DAPT was assessed for safety and efficacy after implantation of a bioabsorbable polymer-based metallic sirolimus eluting stent with a luminal CD34+ antibody coating in patients with ACS. The rationale behind testing such a stent was to use the combination of abluminal release of sirolimus (to prevent neointima formation), and capture of endothelial progenitor cells (to enhance stent re-endothelialization). The cumulative survival free from the primary study end-point of ACM, MI, ST, stroke, TVR, or bleeding for 3-month vs. 12-month DAPT, was $91.7 \%$ vs. $91.5 \%, \mathrm{p}_{\mathrm{ni}}<0.001$. There were, however, concerning safety signals with a higher risk of ACM ( $1.9 \%$ vs. $0.8 \%, p=0.07)$ and ST $(1.2 \%$ vs. $0.4 \% p=0.08)$, with shorter duration of DAPT with no difference in bleeding. Cautious interpretation of these results suggests that though the noninferiority hypothesis was met, the margin of noninferiority was quite generous, and the trend of some ischemic endpoints impoverished the 3-month DAPT group.

\section{2c S-DAPT vs L-DAPT}

Three RCT till date have been published as summarized in Table 4.

TABLE 4 Comparative features of randomized controlled trials(RCTs) for short DAPT(S-DAPT) Vs. long DAPT(L-DAPT)

ACS= acute coronary syndrome; $\mathbf{D M}=$ diabetes mellitus; $\mathbf{1 G}=$ first generation; $\mathbf{2} \mathbf{G}=$ second generation; $\mathbf{M I}=$ myocardial infarction; $\mathbf{T V R}=$ target vessel revascularization; $\mathbf{p}_{\mathrm{ni}}=\mathrm{p}$ value for non-inferiority

PRODIGY $^{54}=$ The PROlonging Dual AntIplatelet Treatment After Grading Stent-Induced Intimal Hyperplasia Study; Short- versus long-term duration of dual-antiplatelet therapy after coronary stenting: a randomized multicenter trial; ITALIC ${ }^{55}=$ Is There A Life for DES after DIscontinuation of Clopidogrel, 6- versus 24-month dual antiplatelet therapy after implantation of drug-eluting stents in patients nonresistant to aspirin; NIPPON ${ }^{56}=$ Dual Antiplatelet Therapy for 6 Versus 18 Months After Biodegradable Polymer Drug-Eluting Stent Implantation

The implications of the data from these trials is certainly influenced by the heterogeneity of patients and of the stents used. These trials were powered to look for difference in bleeding, and due to the low ischemic event rates, any conclusions drawn to qualify the efficacy would be inaccurate. More recently, NIPPON trial ${ }^{56}$ was performed in Japan using bioabsorbable polymer-based DES. This trial tested for non-inferiority of 6month DAPT vs. 18month DAPT, and randomized 3775 patients. The composite primary outcome of ACM, MI, stroke, and major bleeding was similar (1.92\% vs $1.45 \%)$ thus meeting the non-inferiority. However, the margin for such non-inferiority was set wide at $2 \%$ which exceeded the event rate of the experimental arm and the study was prematurely terminated thus raising concerns and these results should be judiciously interpreted. 


\section{2d Standard DAPT vs L-DAPT}

The hypothesis of superiority of L-DAPT in reducing the VLST and other ischemic events in comparison with standard DAPT was tested in four RCTs'. These are comparatively represented in Table 5. In all these trials, event-free patients on 1 year of DAPT were randomized to single antiplatelet therapy (SAPT) vs continuation of DAPT with clopidogrel or prasugrel for varying periods of time.

\section{TABLE 5 Comparative features of randomized controlled trials(RCTs) for standard DAPT Vs. long DAPT(L-} DAPT)

ACS= acute coronary syndrome; $\mathbf{D M}=$ diabetes mellitus; $\mathbf{1 G}=$ first generation; $\mathbf{2 G}$ = second generation; $\mathbf{M I}=$ myocardial infarction; $\mathbf{T V R}=$ target vessel revascularization; $\mathbf{p}_{\mathrm{ni}}=\mathrm{p}$ value for non-inferiority; $\mathbf{S T}=$ stent thrombosis; $\mathbf{M A C C E}=$ major adverse cardiovascular and cerebrovascular events; ISTH= international society of thrombosis and hemostasis

$\mathbf{D A P T}^{28}=$ Dual AntiPlatelet Therapy study, Twelve or 30 months of dual antiplatelet therapy after drug-eluting stents; DES LATE ${ }^{57}=$

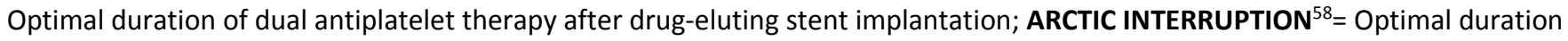
of dual antiplatelet therapy after drug-eluting stent implantation; OPTIDUAL ${ }^{59}=$ Stopping or continuing clopidogrel 12 months after drug-eluting stent placement

DAPT ${ }^{28}$ trial deserves a special mention for being the only trial which was adequately powered for safety and efficacy endpoints, and also providing some significant insights into L-DAPT. In this trial, 9961 patients who were event free after 12 months' of DAPT and compliant to DAPT were randomized to continue DAPT for 30 months' vs SAPT (with aspirin). About $26 \%$ of the participants had ACS and importantly, $47 \%$ of the patients received Everolimus eluting stents (EES) and only clopidogrel (65\%) and prasugrel (35\%) were used as a part of DAPT. In the DAPT group, there was $1 \%$ lower VLST and $1.6 \%$ fewer MACCE events driven by $2 \%$ reduction in rates of MI. These benefits came at a cost of $0.9 \%$ absolute increase in moderate to severe GUSTO (Global Utilization of Streptokinase and t-PA for Occluded Coronary Arteries) bleeding and $2.6 \%$ increase in BARC (Bleeding Academic Research Consortium) 2, 3, or 5 bleeding. At 33 months' follow-up, ACM was higher in the DAPT group ( $2 \%$ vs $1.5 \%, p=0.052$ ). This increase was attributable to bleeding, trauma, and cancer ${ }^{60}$.The authors also interpreted this finding as being due to chance and later it was noted that at baseline, a greater number of patients with a prior history of cancer had been randomly allocated to extended DAPT duration group thus explaining 7 of the 26 deaths in that group. Food and drug administration(FDA) passed a revision refuting an association of increased mortality with extended use of clopidogrel ${ }^{61}$. However, such an increase in fatalities were also observed in other studies ${ }^{62}$ with other agents.

More recently, the hypothesis of 48 months of DAPT with clopidogrel being superior to 12 months of DAPT was tested in OPTIDUAL ${ }^{59}$. The enrollment was prematurely stopped in this trial. Superiority of L-DAPT could not be established as the composite primary end-point of death, $\mathrm{MI}$, stroke, or major hemorrhage were similar in both the arms (5.8\% vs. 7.5\%; HR 0.75; $p=0.17)$. Safety end-point of moderate and severe GUSTO bleeding $(1.9 \%$ vs. $1.7 \%)$ and BARC 2,3 , or 5 bleeding ( $2.6 \%$ vs. $2.9 \%)$ were similar in both groups.

\section{2 e Other RCTs with DAPT duration}

Three other RCTs are worthy of review as they deal with safety and efficacy of DAPT in varied clinical settings.

In CHARISMA ${ }^{63}$ (Clopidogrel for High Atherothormbotic Risk and Ischemic Stabilization, Management, and Avoidance) trial, 15,603 patients with cardiovascular risk factors or a history of vascular disease were randomized to receive DAPT with aspirin and clopidogrel vs SAPT with aspirin. The composite primary 
endpoint of MACCE at 28 months was similar in both groups ( $6.8 \%$ vs $7.3 \%, p=0.22)$, and there was no significant difference in major bleeding. However, there was $1 \%$ risk reduction of MACCE in DAPT group vs SAPT (6.9\% vs 7.9\%; RR 0.88; $p=0.046$ ) when analyzed in the pre-specified group of patients with established cardiovascular disease ${ }^{64}$. Patients with prior $\mathrm{MI}$, stroke, or symptomatic peripheral arterial disease (PAD) derived significant benefit from DAPT $(7.3 \%$ versus $8.8 \%$ HR: $0.83, p=0.01)$ and there was no significant difference in the rate of severe bleeding (1.7\% vs. $1.5 \%$, HR: $1.12 ; p=0.50)$; moderate bleeding was significantly increased (2.0\% vs.1.3\%, HR:1.60; $p=0.004)^{64}$.

In PEGASUS-TIMI 54 ${ }^{29}$ (Prevention of Cardiovascular Events in Patients with Prior Heart Attack Using Ticagrelor Compared to Placebo on a Background of Aspirin-Thrombolysis in Myocardial Infarction 54) trial; 21,162 patients with a prior history of $\mathrm{MI}$ in preceding 1-3 years, were randomized in a double blinded regimen in 1:1:1 into three groups. This trial was designed to test the efficacy of DAPT (Ticagrelor 90mg twice daily or 60 mg twice daily) with aspirin vs SAPT with aspirin. There was 1.2-1.3\% absolute risk reduction (ARR) of MACCE events in DAPT groups' vs SAPT at the cost of 1.2-1.5\% increase in major bleeding. However, there was no excess in fatal bleeding or intracranial hemorrhage. Sub-group analysis of higher risk patients demonstrated more robust benefits. In the diabetic sub-group, there was an ARR of $1.5 \%(p=0.03)^{65}$. Patients with prior vascular disease demonstrated a higher event rate, and despite an increased bleeding risk, there was a nearly $5 \%$ ARR $^{29}$ of ischemic events. Patients with renal disease also had higher event rate but drew more benefit from DAPT therapy with ARR $2.7 \%{ }^{66}$ of ischemic events.

These two trials underpin the ischemic benefit derived from L-DAPT especially in higher risk patients, albeit at the cost of increased bleeding risk. However, it is noteworthy that a majority of these patients had a period of interruption in DAPT after their initial ischemic event. In fact, in PEGASUS pre-specified subgroup analysis, patients with discontinuation period of 1 year or longer before re-initiation of DAPT did not derive any benefit ${ }^{67}$.

In the recently published SENIOR trial, ${ }^{68} 1200$ elderly patients ( $\geq 75$ years of age) with CAD, were randomly assigned to DES or BMS after an intended duration of DAPT (1 month for stable CAD, 6 months for ACS). There was significant reduction in primary composite endpoint of $A C M, M I$, stroke, ischemia driven target lesion revascularization in DES vs BMS [16.4\% vs $11.6 \%, p=0.016 ; R R 0.71]$ thus yielding NNT $=21$. This difference was mainly driven by ischemia driven target lesion revascularization ( $1.7 \%$ vs. $5.9 \%, p=0.0002)$. Net clinical benefit encompassing MACCE and BARC 2-5 bleeding was significantly lower in DES vs BMS [14.4\% vs. $19.2 \%$, $p=0.0239 ;$ RR 0.75]. Interestingly, ST was low and not different between the groups $(0.5 \%$ vs $1.4 \%, p=0.12)$. It has to be noted that the aim of this study was to compare the type of stents but not the DAPT duration. However, it provides valuable information in this group that has not been well represented in prior RCTs'.

\section{2 f Meta-analyses}

The idea of net clinical benefit for the individual patient becomes complicated due the fact that these trials demonstrate reduced ischemic events and increased bleeding with prolongation of DAPT, although with a possible interaction with stent type. This generated a need for meta-analysis of these RCTs'. Many metaanalyses have been performed till date and they have differed significantly in the number of RCTs' included and also their designs ${ }^{69-74}$.

In the largest meta-analysis till date ${ }^{71}$ including 14 RCTs' involving 69,644 patients with ACM as the only primary endpoint, there was no significant difference in mortality with L-DAPT in comparison with S-DAPT ( $H R=1.05,95 \%$ credible interval, 0.96-1.19). However, since this analysis included mixed populations, moderate heterogeneity was present $\left(I^{2}=27 \%\right)$ for the treatment effects. In a recent meta-analysis ${ }^{75}$ of five RCTs' with mean follow up of 2 years or longer involving 20,000 patients, S-DAPT was compared to L-DAPT . 
The primary end point was ST and secondary endpoints were ACM, CVM, MI, TVR, TIMI major bleeding and stroke. Compared to L-DAPT, S-DAPT was associated with higher MI [OR 1.48]. There were no significant differences between groups in all other end points.

\section{Current guidelines}

The current transatlantic guidelines on DAPT usage are summarized in Table 6

\section{TABLE 6 GUIDELINE STATEMENTS ON DAPT USAGE}

$\mathrm{ESC}=$ European society of cardiology focused update on dual antiplatelet therapy in coronary artery disease ${ }^{30}$ $\mathrm{ACC} / \mathrm{AHA}=$ American College of Cardiology/ American Heart association guideline focused update on duration of dual antiplatelet therapy in patients with coronary artery disease ${ }^{2}$

\section{Individualization of therapy}

Decisions regarding DAPT duration are complex and they epitomize the current era of "personalized medicine". The net clinical benefit should be the ultimate goal of this shared decision. DAPT trial offers a decent outlook into this aspect.

In evaluation of net clinical benefit in the DAPT study participants, NNT for benefit from ischemic events was 100 based on 1\% ARR for ST; NNT= 50 based on ARR 2\% for MI. The number needed to harm(NNH) was 111 based on $0.9 \%$ absolute risk of increase (ARI) in bleeding with L-DAPT thus favoring such a strategy ${ }^{28}$. However, when a similar exercise is carried out for the pre-specified patients with second generation DES; NNT $=200$ based on ARR 0.5\% for ST; NNT=91 based on ARR 1.1\% and NNH=83 based on ARI 1.2\% due to bleeding thus disfavoring L-DAPT for prevention of MACCE rate and mortality ${ }^{76}$.

Hence, it's imperative to evaluate the factors conferring ischemic and bleeding risks as listed in Figure 1. Though use of DAPT in reducing ischemic events ${ }^{29,34}$ is well known, it is crucial to recognize the increased risk of bleeding with such therapy ${ }^{29,33}$ which ultimately has adverse prognostic implications ${ }^{77}$ as well.

\section{FIGURE 1 BALANCE BETWEEN ISCHEMIC AND BLEEDING RISKS ${ }^{43,78}$}

ST = Stent thrombosis; $\mathrm{ACS}=$ Acute coronary syndrome; $\mathrm{DM}=$ Diabetes mellitus; $\mathrm{CKD}=$ Chronic kidney disease; $\mathrm{PCl}=$ percutaneous coronary intervention; NSAID= Non-steroidal anti-inflammatory drugs, DAPT= Dual anti platelet therapy

Risk calculators, as endorsed by the most recent guidelines can be an instrumental in making decisions regarding the duration of DAPT ${ }^{30}$. These are summarized in Table 7

\section{TABLE 7 COMPARATIVE FEATURES OF TOOLS FOR RISK ESTIMATION}

$\mathrm{DM}=$ Diabetes mellitus; $\mathrm{MI}=$ Myocardial infarction; $\mathrm{PCl}=$ Percutaneous coronary intervention, $\mathrm{ACS}=$ Acute coronary syndrome; LVEF= Left ventricular ejection fraction

DAPT score was developed to aid clinicians and patients in the assessment of ischemic and bleeding risks. Since the tool was developed from DAPT study data, it can only be applied to patients completing 12 months DAPT uneventfully. This score was internally validated in DAPT study with moderate discrimination [ $C$ statistic, 
$0.70 ; 0.68$ ] and calibrated for both ischemia and bleeding risks (goodness-of-fit $p=0.81, p=0.34)^{78}$. This tool was externally validated in the PROTECT (Patient Related Outcomes with Endeavor versus Cypher stenting) trial cohort ${ }^{79}$.

PRECISE-DAPT (Predicting bleeding Complications In patients undergoing Stent implantation and subsequent Dual Anti Platelet Therapy) study group developed a tool from eight RCTs'. The predictive performance of this tool was assessed in the derivation cohort and validated in 8595 patients from the PLATO trial and 6172 patients from BernPCI registry. In comparison with PARIS bleeding score, this tool demonstrated good discrimination and net reclassification of patients ${ }^{80}$.

These tools have not yet been tested prospectively in a RCT setting, and are by no means perfect or substitutive to clinical judgement ${ }^{81}$.

\section{Triple therapy}

Triple therapy refers to the use of oral anticoagulant (OAC) and DAPT. CAD is a common comorbid condition in patients with atrial fibrillation (AF), and its prevalence was reported as 60-65\% in Medicare beneficiaries ${ }^{82}$. Guidelines recommend assessment of stroke risk by $\mathrm{CHA}_{2} \mathrm{DS}_{2} \mathrm{VASc}$ score in patients with $\mathrm{AF}$, and for scores $\geq 1$ 2 , oral anticoagulant $(\mathrm{OAC})$ is recommended to mitigate risk of thromboembolism commonly manifested as stroke $^{82,83}$. An estimated 5-10\% patients undergoing $\mathrm{PCI}$ have concomitant $\mathrm{AF}$ with a need for OAC ${ }^{84}$. Other clinical situations requiring triple therapy is in patients needing $\mathrm{PCl}$, and with indications for anticoagulation for conditions like deep vein thrombosis(DVT)/pulmonary embolism (PE), mechanical heart valve, left ventricular thrombosis etc. Such therapy comes at a cost of excessive bleeding risk ${ }^{85}$. With the inception of direct oral anticoagulants and potent $\mathrm{P} 2 \mathrm{Y} 12$ inhibitors, clinical decisions on triple therapy remain controversial in the ability to optimize the balance between prevention of stroke and ST without unduly increasing bleeding risk.

The following Table 8 summarizes the salient findings from most recent RCTs.

\section{TABLE 8 EVIDENCE ON TRIPPLE THERAPY}

$\mathrm{BMS}=$ Bare metal stent; $\mathrm{DES}=$ Drug eluting stent; $\mathrm{TIMI}=$ Thrombolyisis in myocardial infarction; $\mathrm{HR}=$ Hazard ratio, $\mathrm{Cl}=\mathrm{Confidence}$ interval; $\mathrm{MI}=$ Myocardial infarction; TVR= target vessel revascularization, $\mathrm{BID}=$ twice daily, $\mathrm{AF}=\mathrm{Atrial}$ fibrillation

WOEST $^{86}=$ What is the Optimal antiplatElet and anticoagulant therapy in patients with oral anticoagulation and coronary StenTing ; PIONEER AF-PCl ${ }^{87}=$ Prevention of Bleeding in Patients with Atrial Fibrillation Undergoing $\mathbf{P C l}$; REDUAL-PCl ${ }^{88}=\mathrm{Dual}$ Antithrombotic Therapy with Dabigatran after PCI in Atrial Fibrillation

In the WOEST trial $^{86}$, the warfarin was evaluated and $70 \%$ patients had AF as the indication for OAC, while 25$30 \%$ had ACS at presentation. The results demonstrated the superiority of dual therapy with warfarin and clopidogrel vs. triple therapy on account of significant reduction in the primary outcome which was any bleeding within 1 year of $\mathrm{PCl}(19.5 \%$ vs. $44.4 \%$; HR $0.36, p<0.001)$ as well as reduction in ACM (2.5\% vs. $6.4 \%$; $\mathrm{p}=0.027)$. The heterogeneity of patients with various indications for $\mathrm{OAC}$ is a limitation of this study.

Subsequently, with the introduction and prevalent use of direct oral anticoagulants, PIONEER AF-PCI ${ }^{87}$ used rivaroxaban and REDUAL-PCl ${ }^{88}$ used dabigatran to evaluate the safety and efficacy of triple therapy exclusively in $\mathrm{AF}$ patients undergoing $\mathrm{PCl}$. 
In the PIONEER AF-PCI ${ }^{87}$, there was 1:1:1 randomization of patients to receive low-dose rivaroxaban (15 $\mathrm{mg}$ daily) + P2Y12 inhibitor for 12 months; very low dose rivaroxaban (2.5 mg twice daily) +DAPT for 1, 6, or 12 months or standard therapy with dose adjusted warfarin + DAPT for 1, 6, 12 months per guideline recommended DAPT duration based on the indication and stent type. There was less bleeding in the rivaroxaban groups vs. warfarin $(17.4 \%$ vs. $26.7 \%$, HR 0.61; $p<0.001)$ without significant difference in MACCE. The rivaroxaban groups had lower re-hospitalization rates in comparison to warfarin $[(34.1 \%$ vs. $41,5 \%, H R$ : $0.77, \mathrm{p}=0.05) ;(31.2 \%$ vs.41.5\%, HR: $0.74, \mathrm{p}=0.01)$ ]. This trial establishes supremacy of rivaroxaban over warfarin in reducing bleeding and re-hospitalizations but it was criticized for the use of $15 \mathrm{mg}$ dose of rivaroxaban which is not approved for use in AF. It has to be emphasized that since the huge majority of the patients received clopidogrel (95\%), this data cannot be extrapolated to the use of other newer and more potent P2Y12 inhibitors as a part of triple therapy regimens.

The results from REDUAL-PCI ${ }^{88}$ were presented at American Heart Association Annual Scientific Sessions (AHA 2017), Anaheim, CA, November 14, 2017. In this trial, AF patients undergoing PCl were randomized in 1:1:1 fashion to dual therapy with dabigatran at a dose of $110 \mathrm{mg}(n=981)$ vs. dual therapy with dabigatran at a dose of $150 \mathrm{mg}(\mathrm{n}=763$ ) vs. triple therapy with warfarin $(n=981)$. In the dual therapy group, participants received clopidogrel or ticagrelor in addition to one of two doses of dabigatran. In the triple therapy group, participants received aspirin plus clopidogrel or ticagrelor in addition to warfarin. The duration of aspirin was 1 month after a BMS and 3 months after a DES. About 52\% patients had ACS, $82 \%$ received DES and $10 \%$ received ticagrelor as the P2Y12 inhibitor. The primary safety outcome, incidence of major or clinically relevant non-major bleeding events was lower in both dabigatran groups vs. triple therapy [( $15.4 \%$ vs. $26.9 \%$, $\left.\mathrm{p}_{\mathrm{ni}}<0.001\right)$; $\left(20.2 \%\right.$ vs $\left.\left.25.7 \%, \mathrm{p}_{\mathrm{ni}}<0.001\right)\right]$. TIMI major bleeding was also lower in dual vs. triple therapy groups. The primary efficacy outcome, incidence of death, MI, stroke, systemic embolism, or unplanned revascularization occurred in $13.7 \%$ of both dual therapy groups vs. $13.4 \%$ of the triple therapy group $\left(p_{n i}=\right.$ 0.005). In the sub-group analysis of patients with ACS (52\%), ticagrelor was associated with higher bleeding compared to clopidogrel, with and without dabigatran.

These several lines of evidence now suggest that it is safe to treat $\mathrm{AF}$ patients who undergo $\mathrm{PCl}$ with anticoagulation and clopidogrel monotherapy. Decisions regarding the type of agents used and duration of such therapy should be individualized based on the risk benefit profile of the patient.

The most recent iteration of ESC guidelines provide evidence based recommendations and possible regimens as listed in the Table 9.

\section{TABLE 9 RECOMMENDED THERAPEUTIC STRATEGIES FOR PATIENTS NEEDING ANTICOAGULATION AND ANTI PLATELET THERAPY}

\section{- $\quad$ Adapted from ESC guideline statement ${ }^{3}$}

There is also an emerging interest in evaluation of the efficacy of combination therapy with OAC and single antiplatelet agent in improving clinical outcome. In the recently published COMPASS $^{89}$ trial, in patients with stable CAD, addition of rivaroxaban to aspirin lowered major vascular events (4\% vs. $6 \% ; \mathrm{HR}: 0.74,95 \% \mathrm{Cl}$ $0.65-0.86, p<0.0001$ ), but increased major bleeding (3\% vs.2\%; HR 1.66, p<0.0001). There was no significant increase in intracranial bleeding or other critical organ bleeding. There was also a significant net benefit in favor of rivaroxaban plus aspirin and deaths were relatively reduced by $23 \%$. Thus, addition of rivaroxaban to aspirin has the potential to substantially reduce morbidity and mortality from CAD. 
In this trial, after a 30-day run in period, patients were randomly assigned (1:1:1) to receive rivaroxaban (2.5 $\mathrm{mg}$ orally twice a day) plus aspirin (100 mg once a day), rivaroxaban alone ( $5 \mathrm{mg}$ orally twice a day), or aspirin alone (100 mg orally once a day). These doses of rivaroxaban are not available in USA for routine use and the data on such combination therapy is still evolving.

\section{Conclusions and future directions}

The data on the duration of DAPT in patients with CAD continues to evolve especially with the availability of newer stent designs and potent antiplatelet agents and newer oral anticoagulants. Novel DES has been shown to be safer than BMS in terms of device related adverse events with both standard DAPT ${ }^{90}$ and S-DAPT ${ }^{91}$. Prolonged DAPT reduces the ischemic events at the cost of bleeding risk, which continues to accrue with longer duration of DAPT. Thus, optimal duration of DAPT remains a moving target. For our readers, we have summarized the future and emerging trials in Table10.

The old adage "there is no free lunch" aptly applies to this clinical dilemma and therefore decisions on DAPT duration require an astute understanding of both the patient's ischemic as well as bleeding risks and "shared decision making" with the patient is recommended.

\section{TABLE 10 COMPARATIVE FEATURES OF PROMINENT FUTURE RANDOMIZED CONTROLLED TRIALS EVALUATING NOVEL DAPT REGIMENS}

$\mathrm{MI}=$ Myocardial infarction; BARC= Bleeding academic research consortium

GLOBAL LEADERS= A Clinical Study comparing two forms of anti-platelet therapy after stent implantation (NCT01813435);

MASTER DAPT= Management of high bleeding risk patients post bioresorbable polymer coated stent implantation with an abbreviated versus prolonged DAPT regimen (NCT03023020); TWILIGHT= Ticagrelor with Aspirin or alone in high-risk patients after coronary intervention (NCT02270242)

\section{Bibliography}

Nabel EG, Braunwald E. A tale of coronary artery disease and myocardial infarction. N Engl J Med 2012; 366: 54-63. Levine GN, Bates ER, Bittl JA, et al. 2016 ACC/AHA guideline focused update on duration of dual antiplatelet therapy in patients with coronary artery disease: A report of the American College of Cardiology/American Heart Association Task Force on Clinical Practice Guidelines. J Thorac Cardiovasc Surg 2016; 152: 1243-75.

Valgimigli M, Bueno H, Byrne RA, et al. 2017 ESC focused update on dual antiplatelet therapy in coronary artery disease developed in collaboration with EACTS: The Task Force for dual antiplatelet therapy in coronary artery disease of the European Society of Cardiology (ESC) and of the European. Eur Heart J 2017; published online Aug. DOI:10.1093/eurheartj/ehx419.

Stefanini GG, Holmes Jr DR. Drug-eluting coronary-artery stents. N Engl J Med 2013; 368: 254-65.

Sigwart U, Puel J, Mirkovitch V, Joffre F, Kappenberger L. Intravascular stents to prevent occlusion and restenosis after transluminal angioplasty. N Engl J Med 1987; 316: 701-6.

Serruys PW, de Jaegere $\mathrm{P}$, Kiemeneij F, et al. A comparison of balloon-expandable-stent implantation with balloon angioplasty in patients with coronary artery disease. Benestent Study Group. N Engl J Med 1994; 331: 489-95. Serruys PW, Unger F, Sousa JE, et al. Comparison of coronary-artery bypass surgery and stenting for the treatment of multivessel disease. N Engl J Med 2001; 344: 1117-24. 
Serruys PW, Strauss BH, Beatt KJ, et al. Angiographic follow-up after placement of a self-expanding coronary-artery stent. $\mathrm{N}$ Engl J Med 1991; 324: 13-7.

Morice MC, Serruys PW, Sousa JE, et al. A randomized comparison of a sirolimus-eluting stent with a standard stent for coronary revascularization. $N$ Engl J Med 2002; 346: 1773-80.

Stone GW, Ellis SG, Cox DA, et al. A polymer-based, paclitaxel-eluting stent in patients with coronary artery disease. $N$ Engl J Med 2004; 350: 221-31.

Stettler C, Wandel S, Allemann S, et al. Outcomes associated with drug-eluting and bare-metal stents: a collaborative network meta-analysis. Lancet (London, England) 2007; 370: 937-48. Kastrati A, Mehilli J, Pache J, et al. Analysis of 14 trials comparing sirolimus-eluting stents with bare-metal stents. $N$ Engl J Med 2007; 356: 1030-9.

Joner M, Nakazawa G, Finn A V, et al. Endothelial cell recovery between comparator polymer-based drug-eluting stents. J Am Coll Cardiol 2008; 52: 333-42.

Alazzoni A, Al-Saleh A, Jolly SS. Everolimus-Eluting versus Paclitaxel-Eluting Stents in Percutaneous Coronary Intervention: Meta-Analysis of Randomized Trials. Thrombosis 2012; 2012: 126369. Joner M, Finn A V, Farb A, et al. Pathology of drug-eluting stents in humans: delayed healing and late thrombotic risk. J Am Coll Cardiol 2006; 48: 193-202. Serruys PW, Garcia-Garcia HM, Onuma Y. From metallic cages to transient bioresorbable scaffolds: change in paradigm of coronary revascularization in the upcoming decade? Eur Heart $J$ 2012; 33: 16-25b. Serruys PW, Onuma Y, Garcia-Garcia HM, et al. Dynamics of vessel wall changes following the implantation of the absorb everolimus-eluting bioresorbable vascular scaffold: a multi-imaging modality study at 6, 12, 24 and 36 months.

Eurolntervention 2014; 9: 1271-84.

Serruys PW, Chevalier B, Dudek D, et al. A bioresorbable everolimus-eluting scaffold versus a metallic everolimus-eluting stent for ischaemic heart disease caused by de-novo native coronary artery lesions (ABSORB II): an interim 1-year analysis of clinical and procedural secondary outcomes from . Lancet (London, England) 2015; 385: 43-54. Byrne RA, Stefanini GF, Capodanno D, et al. Report of an ESC-EAPCI Task Force on the evaluation and use of bioresorbable scaffolds for percutaneous coronary intervention: executive summary. Eurolntervention 2017; published online Sept 25. DOI:EIJ20170912-01 [pii].

Barnathan ES, Schwartz JS, Taylor L, et al. Aspirin and dipyridamole in the prevention of acute coronary thrombosis complicating coronary angioplasty. Circulation 1987; 76: 125-34. Leon MB, Baim DS, Popma JJ, et al. A clinical trial comparing three antithrombotic-drug regimens after coronary-artery stenting. Stent Anticoagulation Restenosis Study Investigators. N Engl J Med 1998; 339: 1665-71. Schomig A, Neumann FJ, Kastrati A, et al. A randomized comparison of antiplatelet and anticoagulant therapy after the placement of coronary-artery stents. N Engl J Med 1996; 334: 1084-9.

McFadden EP, Stabile E, Regar E, et al. Late thrombosis in drug-eluting coronary stents after discontinuation of antiplatelet therapy. Lancet (London, England) 2004; 364: 1519-21. Camenzind E, Steg PG, Wijns W. Stent thrombosis late after implantation of first-generation drug-eluting stents: a cause for concern. Circulation 2007; 115: 1440-55; discussion 1455. (EACTS) TF on MR of the ES of C (ESC) and the EA for C-TS, (EAPCI) EA for PCl, Kolh P, et al. Guidelines on myocardial revascularization. Eur J Cardiothorac Surg 2010; 38 Suppl: S1-52. Stone GW, Maehara A, Lansky AJ, et al. A prospective natural-history study of coronary atherosclerosis. N Engl J Med 2011; 364: 226-35. Windecker S, Kolh P, Alfonso F, et al. 2014 ESC/EACTS guidelines on myocardial revascularization. Eurolntervention 2015; 10: 1024-94.

Mauri L, Kereiakes DJ, Yeh RW, et al. Twelve or 30 months of dual antiplatelet therapy after drug-eluting stents. N Engl J Med 2014; 371: 2155-66. Bonaca MP, Bhatt DL, Cohen M, et al. Long-term use of ticagrelor in patients with prior myocardial rction. N Engl J Med 2015; 372: 1791-800. Valgimigli M, Bueno H, Byrne RA, et al. A Randomized Trial of Bioabsorbable Polymer-Based Metallic DES vs BMS with Short DAPT. Eur J Cardiothorac Surg 2017; published online Aug 26. DOI:10.1093/ejcts/ezx334 [doi]. myocardial infarction: ISIS-2. ISIS-2 (Second International Study of Infarct Survival) Collaborative Group. Lancet (London, England) 1988; 2: 349-60. CAPRIE Steering Committee. Lancet (London, England) 1996; 348: 1329-39. Yusuf S, Zhao F, Mehta SR, et al. Effects of clopidogrel in addition to aspirin in patients with acute coronary syndromes without ST-segment elevation. N Engl J Med 2001; 345: 494-502. 
Mehta SR, Yusuf S, Peters RJ, et al. Effects of pretreatment with clopidogrel and aspirin followed by long-term therapy in patients undergoing percutaneous coronary intervention: the PCl-CURE study. Lancet (London, England) 2001; 358: 527-33. Steinhubl SR, Berger PB, Mann 3rd JT, et al. Early and sustained dual oral antiplatelet therapy following percutaneous coronary intervention: a randomized controlled trial. Jama 2002; 288: 2411-20.

Wiviott SD, Braunwald E, McCabe $\mathrm{CH}$, et al. Prasugrel versus clopidogrel in patients with acute coronary syndromes. $N$ Engl $J$ Med 2007; 357: 2001-15.

Wallentin L, Becker RC, Budaj A, et al. Ticagrelor versus clopidogrel in patients with acute coronary syndromes. N Engl $\mathrm{J}$ Med 2009; 361: 1045-57.

Roe MT, Armstrong PW, Fox KA, et al. Prasugrel versus clopidogrel for acute coronary syndromes without revascularization. N Engl J Med 2012; 367: 1297-309.

Bhatt DL, Stone GW, Mahaffey KW, et al. Effect of platelet inhibition with cangrelor during PCl on ischemic events. N Engl J Med 2013; 368: 1303-13.

Tricoci P, Huang Z, Held C, et al. Thrombin-receptor antagonist vorapaxar in acute coronary syndromes. N Engl J Med 2012; 366: 20-33.

Montalescot G, Wiviott SD, Braunwald E, et al. Prasugrel compared with clopidogrel in patients undergoing percutaneous coronary intervention for ST-elevation myocardial infarction (TRITON-TIMI 38): double-blind, randomised controlled trial. Lancet (London, England) 2009; 373: 723-31.

Sahlen A, Varenhorst C, Lagerqvist B, et al. Outcomes in patients treated with ticagrelor or clopidogrel after acute myocardial infarction: experiences from SWEDEHEART registry. Eur Heart J 2016; 37: 3335-42.

Levine GN, Bates ER. It Is Time to End the Dualistic Short Versus Long Duration of Dual Antiplatelet Therapy Debates. Circulation 2017; 135: 2451-3.

Costa F, Windecker S, Valgimigli M. Dual Antiplatelet Therapy Duration: Reconciling the Inconsistencies. Drugs 2017; 77: 1733-54.

Gwon HC, Hahn JY, Park KW, et al. Six-month versus 12-month dual antiplatelet therapy after implantation of drug-eluting stents: the Efficacy of Xience/Promus Versus Cypher to Reduce Late Loss After Stenting (EXCELLENT) randomized, multicenter study. Circulation 2012; 125: 505-13.

Kim BK, Hong MK, Shin DH, et al. A new strategy for discontinuation of dual antiplatelet therapy: the RESET Trial (REal Safety and Efficacy of 3-month dual antiplatelet Therapy following Endeavor zotarolimus-eluting stent implantation). J Am Coll Cardiol 2012; 60: 1340-8.

Feres F, Costa RA, Abizaid A, et al. Three vs twelve months of dual antiplatelet therapy after zotarolimus-eluting stents: the OPTIMIZE randomized trial. Jama 2013; 310: 2510-22.

Colombo A, Chieffo A, Frasheri A, et al. Second-generation drug-eluting stent implantation followed by 6- versus 12-month dual antiplatelet therapy: the SECURITY randomized clinical trial. J Am Coll Cardiol 2014; 64: 2086-97.

Schulz-Schupke S, Byrne RA, Ten Berg JM, et al. ISAR-SAFE: a randomized, double-blind, placebo-controlled trial of 6 vs. 12 months of clopidogrel therapy after drug-eluting stenting. Eur Heart J 2015; 36: 1252-63.

Han Y, Xu B, Xu K, et al. Six Versus 12 Months of Dual Antiplatelet Therapy After Implantation of Biodegradable Polymer Sirolimus-Eluting Stent: Randomized Substudy of the I-LOVE-IT 2 Trial. Circ Interv 2016; 9: e003145.

Hong SJ, Shin DH, Kim JS, et al. 6-Month Versus 12-Month Dual-Antiplatelet Therapy Following Long Everolimus-Eluting Stent Implantation: The IVUS-XPL Randomized Clinical Trial. JACCCardiovascular Interv 2016; 9: 1438-46.

Kedhi E, Fabris E, van der Ent M, et al. A prospective, randomized, open-label trial of 6-month versus 12-month dual antiplatelet therapy after drug-eluting stent implantation in ST-elevation myocardial infarction: Rationale and design of the 'DAPT-STEMI trial'. Am Heart J 2017; 188: 11-7.

Camaro C, Damen SAJ, Brouwer MA, et al. Randomized evaluation of short-term dual antiplatelet therapy in patients with acute coronary syndrome treated with the COMBO dual therapy stent: rationale and design of the REDUCE trial. Am Heart J 2016; 178: 37-44.

Valgimigli M, Campo G, Monti M, et al. Short- versus long-term duration of dual-antiplatelet therapy after coronary stenting: a randomized multicenter trial. Circulation 2012; 125: 2015-26.

Gilard M, Barragan P, Noryani AAL, et al. 6- versus 24-month dual antiplatelet therapy after implantation of drug-eluting stents in patients nonresistant to aspirin: the randomized, multicenter ITALIC trial. J Am Coll Cardiol 2015; 65: 777-86. Nakamura M, lijima R, Ako J, et al. Dual Antiplatelet Therapy for 6 Versus 18 Months After Biodegradable Polymer DrugEluting Stent Implantation. JACCCardiovascular Interv 2017; 10: 1189-98.

Lee CW, Ahn JM, Park DW, et al. Optimal duration of dual antiplatelet therapy after drug-eluting stent implantation: a randomized, controlled trial. Circulation 2014; 129: 304-12.

Collet JP, Silvain J, Barthelemy O, et al. Dual-antiplatelet treatment beyond 1 year after drug-eluting stent implantation (ARCTIC-Interruption): a randomised trial. Lancet (London, England) 2014; 384: 1577-85.

Helft G, Steg PG, Le Feuvre C, et al. Stopping or continuing clopidogrel 12 months after drug-eluting stent placement: the OPTIDUAL randomized trial. Eur Heart J 2016; 37: 365-74. 
60 Mauri L, Elmariah S, Yeh RW, et al. Causes of late mortality with dual antiplatelet therapy after coronary stents. Eur Heart J 2016; 37: 378-85.

61 Professionals HC. Plavix ( clopidogrel ): Drug Safety Communication - Long-term Treatment Does Not Change Risk of. 2015; : $1-2$.

62 Costa F, Valgimigli M. Long-Term Use of Ticagrelor in Patients with Prior Myocardial Infarction. N Engl J Med 2015; 373: 1271-2.

63 Bhatt DL, Fox KA, Hacke W, et al. Clopidogrel and aspirin versus aspirin alone for the prevention of atherothrombotic events. N Engl J Med 2006; 354: 1706-17. Bhatt DL, Flather MD, Hacke W, et al. Patients with prior myocardial infarction, stroke, or symptomatic peripheral arterial disease in the CHARISMA trial. J Am Coll Cardiol 2007; 49: 1982-8.

Bhatt DL, Bonaca MP, Bansilal S, et al. Reduction in Ischemic Events With Ticagrelor in Diabetic Patients With Prior Myocardial Infarction in PEGASUS-TIMI 54. J Am Coll Cardiol 2016; 67: 2732-40.

Magnani G, Storey RF, Steg G, et al. Efficacy and safety of ticagrelor for long-term secondary prevention of atherothrombotic events in relation to renal function: insights from the PEGASUS-TIMI 54 trial. Eur Heart J 2016; 37: 400-8. Bonaca MP, Bhatt DL, Steg PG, et al. Ischaemic risk and efficacy of ticagrelor in relation to time from P2Y12 inhibitor withdrawal in patients with prior myocardial infarction: insights from PEGASUS-TIMI 54. Eur Heart J 2016; 37: $1133-42$. Varenne $\mathrm{O}$, Cook S, Sideris $\mathrm{G}$, et al. Drug-eluting stents in elderly patients with coronary artery disease (SENIOR): a randomised single-blind trial. Lancet (London, England) 2018; 391: 41-50. Valgimigli M, Park SJ, Kim HS, et al. Benefits and risks of long-term duration of dual antiplatelet therapy after drug-eluting stenting: a meta-analysis of randomized trials. Int J Cardiol 2013; 168: 2579-87. Genereux P, Rutledge DR, Palmerini T, et al. Stent Thrombosis and Dual Antiplatelet Therapy Interruption With EverolimusEluting Stents: Insights From the Xience V Coronary Stent System Trials. Circ Interv 2015; 8: 10.1161/CIRCINTERVENTIONS.114.001362.

Elmariah S, Mauri L, Doros G, et al. Extended duration dual antiplatelet therapy and mortality: a systematic review and metaanalysis. Lancet (London, England) 2015; 385: 792-8.

Giustino G, Baber U, Sartori S, et al. Duration of dual antiplatelet therapy after drug-eluting stent implantation: a systematic review and meta-analysis of randomized controlled trials. J Am Coll Cardiol 2015; 65: 1298-310.

Palmerini T, Benedetto U, Bacchi-Reggiani L, et al. Mortality in patients treated with extended duration dual antiplatelet therapy after drug-eluting stent implantation: a pairwise and Bayesian network meta-analysis of randomised trials. Lancet (London, England) 2015; 385: 2371-82.

Navarese EP, Andreotti F, Schulze V, et al. Optimal duration of dual antiplatelet therapy after percutaneous coronary intervention with drug eluting stents: meta-analysis of randomised controlled trials. BMJ 2015; 350: h1618. Sharma A, Agrawal S, Garg A, Vallakati A, Lavie CJ, Helft G. Duration of dual antiplatelet therapy following drug-eluting stent implantation: A systemic review and meta-analysis of randomized controlled trials with longer follow up. Catheter Cardiovasc Interv 2017; 90: 31-7. Hermiller JB, Krucoff MW, Kereiakes DJ, et al. Benefits and Risks of Extended Dual Antiplatelet Therapy After EverolimusEluting Stents. JACCCardiovascular Interv 2016; 9: 138-47. Mehran R, Pocock SJ, Nikolsky E, et al. A risk score to predict bleeding in patients with acute coronary syndromes. J Am Coll Cardiol 2010; 55: 2556-66. Tahir UA, Yeh RW. Individualizing dual antiplatelet therapy duration after percutaneous coronary intervention: from randomized control trials to personalized medicine. Expert Rev Cardiovasc Ther 2017; 15: 681-93. Yeh RW, Secemsky EA, Kereiakes DJ, et al. Development and Validation of a Prediction Rule for Benefit and Harm of Dual Antiplatelet Therapy Beyond 1 Year After Percutaneous Coronary Intervention. Jama 2016; 315: 1735-49. Costa $\mathrm{F}$, van Klaveren $\mathrm{D}$, James $\mathrm{S}$, et al. Derivation and validation of the predicting bleeding complications in patients undergoing stent implantation and subsequent dual antiplatelet therapy (PRECISE-DAPT) score: a pooled analysis of individual-patient datasets from clinical trials. Lancet (London, England) 2017; 389: 1025-34. Capodanno D, Angiolillo DJ. Tailoring duration of DAPT with risk scores. Lancet (London, England) 2017; 389: 987-9. January CT, Wann LS, Alpert JS, et al. 2014 AHA/ACC/HRS guideline for the management of patients with atrial fibrillation: executive summary: a report of the American College of Cardiology/American Heart Association Task Force on practice guidelines and the Heart Rhythm Society. Circulation 2014; 130: 2071-104. Kirchhof P, Benussi S, Kotecha D, et al. 2016 ESC Guidelines for the management of atrial fibrillation developed in collaboration with EACTS. Eur Heart J 2016; 37: 2893-962.

Wiviott SD, Braunwald E, Angiolillo DJ, et al. Greater clinical benefit of more intensive oral antiplatelet therapy with prasugrel in patients with diabetes mellitus in the trial to assess improvement in therapeutic outcomes by optimizing platelet inhibition with prasugrel-Thrombolysis in Myocardial I. Circulation 2008; 118: 1626-36. Gurwitz JH, Goldberg RJ. Age-based exclusions from cardiovascular clinical trials: implications for elderly individuals (and for 
all of us): comment on 'the persistent exclusion of older patients from ongoing clinical trials regarding heart failure'. Arch Intern Med 2011; 171: 557-8.

86 Dewilde WJM, Oirbans T, Verheugt FWA, et al. Use of clopidogrel with or without aspirin in patients taking oral anticoagulant therapy and undergoing percutaneous coronary intervention: an open-label, randomised, controlled trial. Lancet (London, England) 2013; 381: 1107-15.

87 Gibson CM, Mehran R, Bode C, et al. Prevention of Bleeding in Patients with Atrial Fibrillation Undergoing PCI. N Engl J Med 2016; 375: 2423-34.

88 Cannon CP, Bhatt DL, Oldgren J, et al. Dual Antithrombotic Therapy with Dabigatran after PCl in Atrial Fibrillation. N Engl J Med 2017; 377: 1513-24.

89 Connolly SJ, Eikelboom JW, Bosch J, et al. Rivaroxaban with or without aspirin in patients with stable coronary artery disease: an international, randomised, double-blind, placebo-controlled trial. Lancet (London, England) 2017; published online Nov. DOI:10.1016/S0140-6736(17)32458-3.

90 Bonaa KH, Mannsverk J, Wiseth R, et al. Drug-Eluting or Bare-Metal Stents for Coronary Artery Disease. N Engl J Med 2016; 375: 1242-52.

91 Urban P, Meredith IT, Abizaid A, et al. Polymer-free Drug-Coated Coronary Stents in Patients at High Bleeding Risk. N Engl J Med 2015; 373: 2038-47. 